\title{
Análise de diferentes doses de nitrogênio e espaçamento em milheto no norte do Tocantins
}

\author{
João Vidal de Negreiros Neto \\ Antônio Clementino dos Santos* \\ Raimundo Laerton de Lima Leite \\ Rossini Sôffa da Cruz \\ Universidade Federal do Tocantins, Campus de Araguaína \\ BR 153, km 112, Caixa Postal 132, Zona Rural, CEP 77804-970, Araguaína-TO, Brasil \\ *Autor para correspondência \\ clementino@uft.edu.br
}

Submetido em 17/11/2009

Aceito para publicação em 16/08/2010

\section{Resumo}

O experimento foi conduzido na Escola de Medicina Veterinária e Zootecnia do Campus de Araguaína, da Universidade Federal do Tocantins, com o objetivo de verificar os efeitos de quatros níveis de adubação nitrogenada $\left(0,30,60\right.$ e $\left.120 \mathrm{~kg} \cdot \mathrm{ha}^{-1}\right)$, e quatro diferentes espaçamentos $(0,25,0,35,0,45$ e $0,65 \mathrm{~m})$ sobre a altura de plantas e produção de biomassa e matéria seca da cultura do milheto - Pennisetum glaucum L. O delineamento experimental utilizado foi o de blocos casualizados, com dezesseis tratamentos, em fatorial $4 \mathrm{x}$ 4 e quatro repetições, totalizando 64 unidades experimentais, com $9 \mathrm{~m}^{2}$ cada. As alturas de plantas, o acúmulo de biomassa e de massa seca foi avaliado semanalmente a partir de 14 dias após a semeadura. Na maior dose de nitrogênio $\left(120 \mathrm{~kg} \cdot \mathrm{ha}^{-1}\right)$ as produções de massa seca foram superiores às demais doses. Não houve diferença significativa entre os espaçamentos utilizados e a altura de plantas em todos os estágios fenológicos avaliados. No entanto, a produção de massa seca foi diferente, sendo que o espaçamento de $0,25 \mathrm{~m}$ foi o que apresentou maiores valores, seguido pelos espaçamentos $0,35 \mathrm{~m}, 0,45 \mathrm{~m}$ e $0,65 \mathrm{~m}$.

Unitermos: manejo,Pennisetum glaucum, produtividade

\section{Abstract}

Analysis of the effects of different nitrogen doses and spacing on millet in northern Tocantins. The experiment was conducted at the School of Veterinary Medicine and Zootechnils, Federal University of Tocantins, in order to verify the effects of four nitrogenous manuring levels $\left(0,30,60\right.$ and $\left.120 \mathrm{~kg}^{-h^{-1}}\right)$ and different spacings $(0.25,0.35,0.45$ and $0.65 \mathrm{~m})$ on plant size, biomass and dry mass in the production of millet (Pennisetum glaucum L.). The experimental design consisted of complete blocks, with 16 treatments and four replications in a $4 \times 4$ factorial arrangement to form a total of 64 experimental units, each of $9 \mathrm{~m}^{2}$. The plant sizes and accumulation of biomass and dry matter were estimated weekly starting from 14 days after seeding. For the highest nitrogen level $\left(120 \mathrm{~kg} \cdot \mathrm{ha}^{-1}\right)$, the biomass and dry mass production were also the highest. There were no significant differences between the spacings utilized and the plant sizes at any of the phenological stages evaluated. However, the dry mass production was distinct, considering that the spacing of $0.25 \mathrm{~m}$ presented the highest values, followed by the $0.35 \mathrm{~m}, 0.45 \mathrm{~m}$ and $0.65 \mathrm{~m}$ spacings.

Key words: management, Pennisetum glaucum, productivity 


\section{Introdução}

Nas regiões tropicais, uma alternativa capaz de intensificar a produção de rebanhos leiteiros e de corte é a utilização de áreas de pastagens cultivadas constituídas por espécies de elevado potencial forrageiro. Neste contexto, o milheto, Pennisetum glaucum (L) - Poacea de ciclo anual, tem se sobressaído por suas características de alta produção e boa qualidade nutricional alcançados nos períodos mais quentes do ano (Degenhart et al., 1995; Heringer, 1995). Além disso, essa espécie é indicada como forrageira para sucessão ao cultivo de verão, nas regiões subtropical e tropical do Brasil.

A área plantada com a cultura do milheto no Brasil é de cerca de 2,1 milhões de hectares (Bonamigo, 1999), sendo que ele é mais cultivado onde se pratica o plantio direto. Nas regiões de Cerrado, a área plantada de milheto tem aumentado nos últimos 15 anos, devido a sua resistência à seca (Pitol et al., 1996), pelo potencial de cobertura do solo oferecido para a prática do plantio direto e para o uso como forrageiro na pecuária. Segundo Costa (1992), a planta é capaz de emitir raízes primárias até 3,6m de profundidade. Por apresentar um sistema radicular volumoso e profundo, propicia melhor uso da água do solo, bem como a reciclagem de nutrientes (Kichel e Macedo, 1996; Netto, 1998).

Apesar de suas características de bom desempenho em regiões secas e de baixas fertilidades, para se obter elevadas produtividades é necessária à utilização de variedades melhoradas, de adubação e de tratos culturais adequados. A exigência nutricional da planta torna-se mais intensa com o início da fase reprodutiva, sendo mais crítica por ocasião da formação das sementes, quando considerável quantidade de nutrientes é translocada. (Carvalho e Nakagawa, 2000).

Dentre os nutrientes, o nitrogênio é o mais exigido pela planta. A adubação inadequada deste nutriente impede que a planta expressa seu potencial produtivo. O manejo inadequado de adubação nitrogenada é um dos principais problemas que contribui para a baixa produtividade das plantas forrageiras tropicais como o milheto. Formas de aplicação de adubos para as diferentes épocas de plantio ainda são discutidas, porém, é certo que a maior disponibilidade de nitrogênio $(\mathrm{N})$, nos estádios mais tardios do ciclo da planta promove um incremento na taxa fotossintética, ocorrendo assim maior assimilação de carbono e, portanto, maior acúmulo de carboidratos que serão transportados para as sementes (Carvalho e Nakagawa, 1980).

Para se atingir elevadas produtividades, além da adequação da adubação é necessário definir de modo adequado a época, a densidade e os espaçamentos. O plantio pode ser em linha ou a lanço, mas em ambos há necessidade de definição ou estabelecimento da época e da densidade de plantio, da quantidade de sementes, do espaçamento, do sistema de semeadura, da profundidade de plantio, dentre outros fatores não menos importantes, como manejo de plantas daninhas, de pragas e doenças, da fertilidade e o manejo de água como no caso de produção de sementes.

O espaçamento evidencia uma característica interessante do milheto, que é a plasticidade de desenvolver-se conforme o espaço de que dispõe. Diversas são as recomendações sobre o melhor espaçamento para o milheto, no entanto, são raros os trabalhos que avaliam a produção de grãos sob diferentes espaçamentos para uma mesma densidade de semeadura. O plantio geralmente é realizado por semeadura, não se adotando espaçamento definido.

Com base no exposto, o presente trabalho tem como objetivo avaliar o desenvolvimento e acúmulo de matéria seca do milheto para silagem em função da adubação nitrogenada e espaçamentos na região norte do Estado do Tocantins.

\section{Material e Métodos}

O experimento foi conduzido na Fazenda da Escola de Veterinária e Zootecnia do Campus de Araguaína, da Universidade Federal do Tocantins. Aárea localiza-se entre as seguintes coordenadas geográficas: $07^{\circ} 06^{\prime} 46,0^{\prime \prime} \mathrm{S}, 48^{\circ} 11^{\prime} 41,1$ ' $\mathrm{O}$, com altitude de $236 \mathrm{~m}$.. O clima da região é classificado como Aw (quente e úmido), com chuvas de outubro a maio, com precipitação média anual de $1802 \mathrm{~mm}$. O solo da área foi classificado como Neossolo Quatzarênco Órtico típico.

O delineamento experimental utilizado foi o de blocos casualizados, em fatorial $4 \times 4$ com 4 repetições, 
sendo quatros níveis crescentes de $\mathrm{N}(0,30,60$ e $\left.120 \mathrm{~kg} \cdot \mathrm{ha}^{1}\right)$, e quatros diferentes espaçamentos $(0,25$; 0,$35 ; 0,45$ e $0,65 \mathrm{~m}$ entre linhas). Totalizando-se 64 unidades experimentais, com $9 \mathrm{~m}^{2}$ cada. Realizou-se a semeadura em sulcos com aproximadamente 2 a $3 \mathrm{~cm}$ de profundidade, utilizando 20 a 30 sementes por metro linear, sendo utilizado a cultivar BRS 1501.

A semeadura foi efetuada em 03 de abril de 2006, sendo o preparo do solo realizado de forma convencional e a adubação de $\mathrm{P}$ e $\mathrm{K}$ foi aplicado de acordo com a análise do solo $\left(42 \mathrm{~kg} \cdot \mathrm{ha}^{-1}\right.$ de $\mathrm{P}_{2} \mathrm{O}_{5}$, na forma de superfosfato simples e $24 \mathrm{~kg} \cdot \mathrm{ha}^{-1}$ de $\mathrm{K}_{2} \mathrm{O}$, na forma de cloreto de potássio). Os níveis crescentes de nitrogênio foram aplicados somente em cobertura, na forma de uréia, sendo fracionada em duas etapas: a primeira 15 dias (três folhas expandidas) e a segunda 25 dias após a semeadura (DAS).

As alturas das plantas, o acúmulo de biomassa e de MS foi avaliado semanalmente a partir de 14 dias após a semeadura, a partir de uma medida linear de 0,50m. Como houve uma medida de espaçamento diferente para cada tratamento, a área colhida por unidade experimental também variou $\left(0,25 \mathrm{~m} \times 0,50 \mathrm{~m}=0,125 \mathrm{~m}^{2} ; 0,35 \mathrm{~m} \times\right.$ $0,50 \mathrm{~m}=0,175 \mathrm{~m}^{2} ; 0,4 \mathrm{~m} \times 0,50 \mathrm{~m}=0,225 \mathrm{~m}^{2}$ e $0,65 \mathrm{~m} \times$ $\left.0,50 \mathrm{~m}=0,325 \mathrm{~m}^{2}\right)$.

As medições das alturas das plantas foram efetuadas com a folha estendida na altura média do dossel. $\mathrm{O}$ corte das plantas para amostragem era feito à altura de $5 \mathrm{~cm}$ do solo.

As medidas semanais de altura eram seguidas de cortes das plantas para conhecimento de MS com medidas subseqüentes feitas em outras plantas.

Foi utilizado o programa estatístico ESTAT, sendo que para a determinação das equações que melhor se ajustaram, os dados foram submetidos à análise de variância e regressão (quantitativo) e aplicado o teste de Tukey (qualitativo), ao nível de 5\% de significância.

\section{Resultados e Discussão}

As alturas das plantas não apresentaram diferenças significativas entre os diferentes espaçamentos (Tabela 1). Porém, a produção de MS no espaçamento mais adensado $(0,25 \mathrm{~m}$ entre linhas) foi significativamente diferente dos demais tratamentos (Tabela 2 e Figura 1b), apresentando diferença significativa entre os demais espaçamentos (Tabela 2, Figura 1c).

O regime hídrico, durante a condução do experimento, apresentou maior concentração pluviométrica, pois no período de 64 dias (do plantio ao último corte) recebeu um total de precipitação pluviométrica de $458,3 \mathrm{~mm}$, não havendo chuva nos últimos 10 dias de experimento. Tal condição pode ter influenciado em perdas de $\mathrm{N}$ por lixiviação, em virtude das aplicações de $\mathrm{N}$ terem acontecido nos dias de grandes precipitações. Ao mesmo tempo, o nitrogênio aplicado na forma de uréia, muito volátil, pode ter melhorado o aproveitamento do $\mathrm{N}$ num ambiente de solo úmido.

Para fontes nitrogenadas como a uréia, essa forma de distribuição do $\mathrm{N}$ mineral (a lanço) pode resultar em perdas expressivas por volatilização, principalmente quando em solos de textura arenosa e de teor de matéria orgânica baixa, cuja capacidade de retenção de amônia é limitada (Rodrigues e Kiehl, 1986). Além disso, Almeida et al. (2000) sugerem que as respostas à aplicação do N podem ser afetadas por qualquer tipo e nível de estresse devido ao ciclo curto da cultura, ao sistema radicular

TABELA 1: Altura das plantas de milheto em função do espaçamento.

\begin{tabular}{ccccccccc}
\hline \multirow{2}{*}{$\begin{array}{c}\text { Espaçamento } \\
(\mathbf{m})\end{array}$} & \multicolumn{8}{c}{ Tempo, DAS } \\
\cline { 2 - 9 } & $\mathbf{1 4}$ & $\mathbf{2 1}$ & $\mathbf{2 8}$ & $\mathbf{3 5}$ & $\mathbf{4 2}$ & $\mathbf{4 9}$ & $\mathbf{5 6}$ & $\mathbf{6 3}$ \\
\hline 0,25 & $7,2 \mathrm{a}^{*}$ & $15,9 \mathrm{a}$ & $32,4 \mathrm{a}$ & $45,4 \mathrm{a}$ & $85,6 \mathrm{a}$ & $133,1 \mathrm{a}$ & $157,4 \mathrm{a}$ & $167,2 \mathrm{a}$ \\
0,35 & $7,2 \mathrm{a}$ & $16,0 \mathrm{a}$ & $28,1 \mathrm{a}$ & $44,0 \mathrm{a}$ & $80,5 \mathrm{a}$ & $135,6 \mathrm{a}$ & $158,4 \mathrm{a}$ & $170,6 \mathrm{a}$ \\
0,45 & $7,4 \mathrm{a}$ & $15,3 \mathrm{a}$ & $29,8 \mathrm{a}$ & $42,5 \mathrm{a}$ & $74,6 \mathrm{a}$ & $124,5 \mathrm{a}$ & $145,3 \mathrm{a}$ & $160,6 \mathrm{a}$ \\
0,65 & $7,4 \mathrm{a}$ & $15,6 \mathrm{a}$ & $29,7 \mathrm{a}$ & $38,6 \mathrm{a}$ & $71,9 \mathrm{a}$ & $132,5 \mathrm{a}$ & $151,7 \mathrm{a}$ & $177,5 \mathrm{a}$ \\
\hline
\end{tabular}

*Médias seguidas pela mesma letra na coluna não diferem entre si ao nível de $1 \%$ de probabilidade pelo teste Tukey. 
TABELA 2: Acúmulo de massa seca de milheto em função do espaçamento.

\begin{tabular}{ccccccccc}
\hline \multirow{2}{*}{$\begin{array}{c}\text { Espaçamento } \\
(\mathbf{m})\end{array}$} & \multicolumn{7}{c}{ Massa Seca (mg.ha-1) } \\
\cline { 2 - 9 } & $\mathbf{1 4}$ & $\mathbf{2 1}$ & $\mathbf{2 8}$ & $\mathbf{3 5}$ & $\mathbf{4 2}$ & $\mathbf{4 9}$ & $\mathbf{5 6}$ & $\mathbf{6 3}$ \\
\cline { 2 - 9 } & $0,02 \mathrm{a}^{*}$ & $0,13 \mathrm{a}$ & $0,5 \mathrm{a}$ & $0,8 \mathrm{a}$ & $2,8 \mathrm{a}$ & $4,6 \mathrm{a}$ & $8,0 \mathrm{a}$ & $8,8 \mathrm{a}$ \\
0,25 & $0,01 \mathrm{~b}$ & $0,10 \mathrm{~b}$ & $0,4 \mathrm{ab}$ & $0,6 \mathrm{ab}$ & $1,7 \mathrm{~b}$ & $4,7 \mathrm{a}$ & $6,7 \mathrm{a}$ & $6,8 \mathrm{~b}$ \\
0,35 & $0,01 \mathrm{~b}$ & $0,07 \mathrm{c}$ & $0,3 \mathrm{bc}$ & $0,5 \mathrm{~b}$ & $1,3 \mathrm{~b}$ & $3,2 \mathrm{~b}$ & $5,0 \mathrm{~b}$ & $5,4 \mathrm{bc}$ \\
0,45 & $0,009 \mathrm{~b}$ & $0,04 \mathrm{~d}$ & $0,2 \mathrm{c}$ & $0,3 \mathrm{c}$ & $1,2 \mathrm{~b}$ & $3,2 \mathrm{~b}$ & $4,2 \mathrm{~b}$ & $4,2 \mathrm{c}$ \\
0,65 & &
\end{tabular}

*Médias seguidas pela mesma letra na coluna não diferem entre si ao nível de 1\% de probabilidade pelo teste Tukey.

pouco desenvolvido, à cultivar utilizada, às condições de clima e à situação do solo no local de semeadura, como, por exemplo, cultura anterior, teor de matéria orgânica, compactação do solo, textura e irrigação, o que permite respostas diferenciadas de um ano para outro até mesmo na própria área.

Apesar de as maiores plantas, ao final do experimento (Tabela 1, Figura 1a), terem sido as que receberam tratamento com $60 \mathrm{~kg} \cdot \mathrm{ha}^{-1}$ de $\mathrm{N}$ e espaçamento de $0,65 \mathrm{~m}$ entre as linhas, não houve diferença significativa entre os tratamentos, a 5\% de probabilidade de ocorrência de erros pelo teste Tukey, exceto no tratamento que não recebeu nitrogênio, diferindo significativamente dos demais. Os diferentes níveis de espaçamento não interferiram no crescimento das plantas (Tabela 1, Figura 1a). $\mathrm{O} N$ atmosférico pode ter contribuído para o aporte do elemento no sistema solo-planta, sendo necessário uma pequena dosagem $\left(30 \mathrm{~kg} \cdot \mathrm{ha}^{-1}\right)$ para se ter uma resposta considerável.

Trabalhos conduzidos por Hanna e Wright (1995) verificaram que a época de semeadura influencia no rendimento e na altura da planta. A média de altura sugerida pelos autores, para semeadura no mês de abril, é de $1,63 \mathrm{~m}$. Na Tabela 1, observa-se que as médias alcançadas superaram tal expectativa exceto no tratamento com $0,45 \mathrm{~m}$ de espaçamento.

Houve efeito significativo para altura de plantas e doses de nitrogênio, a partir de 28 dias após a semeadura. A não diferença antes dos 28 dias é atribuída ao fato de que a adubação nitrogenada de cobertura foi realizada somente aos 21 dias após a semeadura, não interferindo, portanto, nos primeiros dois estágios medidos (14 e 21 DAS). Na maior dose de nitrogênio (120kg.ha-1) o acúmulo final de biomassa (Figura 1b) e de massa seca foram superiores às demais doses (Tabela 2 e Figura 1c). Observa-se ainda que, à medida que aumentou o nível de $\mathrm{N}$, houve um aumento linear na produção de massa seca.

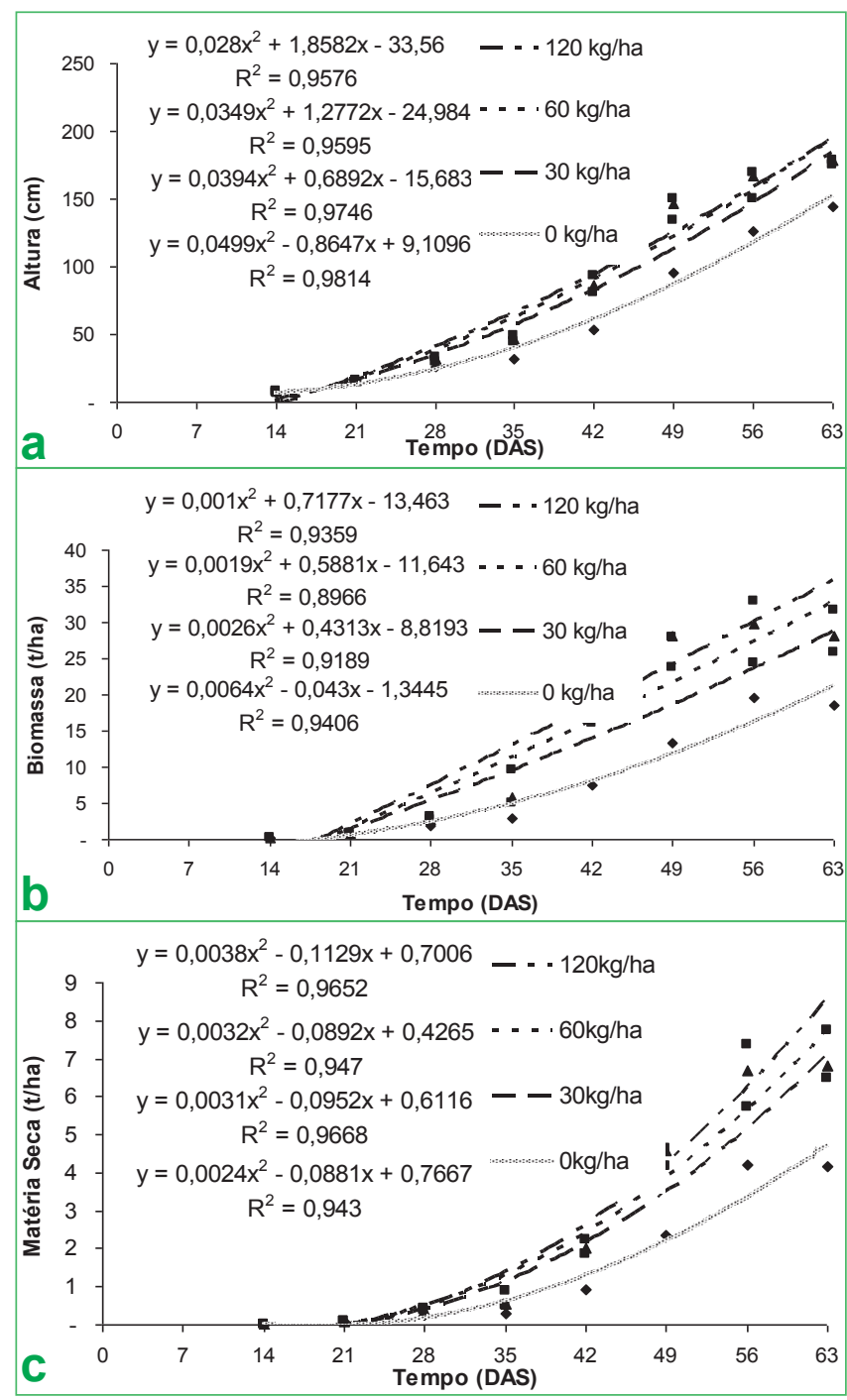

FIGURA 1: Altura das plantas de milheto (a), produção de biomassa (b) e produção de MS (c), em função de níveis de adubação. 
Não houve diferença significativa entre os espaçamentos utilizados e a altura de plantas em todos os estágios fenológicos avaliados (Tabela 1). No entanto, as produções de biomassa e matéria seca diferiram, sendo que o espaçamento de $0,25 \mathrm{~m}$ foi o que apresentou maiores valores, seguido pelos espaçamentos $0,35 \mathrm{~m}$, $0,45 \mathrm{~m}$ e $0,65 \mathrm{~m}$. O mesmo ocorreu com a produção de grãos, isto é, no espaçamento mais estreito observou-se o melhor rendimento.

Outros trabalhos avaliando os espaçamentos de 38 e $76 \mathrm{~cm}$ para controlar o crescimento de plantas daninhas na cultura do milheto também mostraram maior rendimento de grãos no espaçamento mais estreito. Woodruff (1995), citado por Andrews et al. (1996), trabalhando com diferentes espaçamentos para a cultura do milheto, verificou que o espaçamento menor proporcionou maior rendimento de grãos.

Verifica-se pelos resultados e para as condições edafoclimáticas da região, a maior altura de plantas (cm) se deu com os tratamentos que receberam doses de $\mathrm{N}$ de $60 \mathrm{~kg} \cdot \mathrm{ha}^{-1}$ e $120 \mathrm{~kg} \cdot \mathrm{ha}^{-1}$, com espaçamentos de $0,65 \mathrm{~m}$ entre fileiras. $\mathrm{O}$ maior acúmulo de biomassa e MS ocorreu nos tratamentos com aplicações de $120 \mathrm{~kg}$. ha $^{-1}$ de $\mathrm{N}$, e com espaçamento mais adensado $(0,25 \mathrm{~m}$ entre fileiras).

\section{Agradecimentos}

Os autores agradecem a Universidade Federal do Tocantins, ao Programa de Pós-Graduação em Ciência Animal Tropical - Campus de Araguaína, ao $\mathrm{CNPq}$ (pelas bolsas concedidadas) e ao Projeto Centro Tecnológico em Bovinocultura pelo esforço desprendido em atender a alguns pleitos financeiros e estruturais.

\section{Referências}

Alcântara, P. B. 1998. Plantas forrageiras: gramíneas e leguminosas. Nobel, São Paulo, Brasil, 162pp.

Almeida, C.; Carvalho, M. A. C.; Arf, O.; Sá, M. E.; Buzetti, S. 2000. Uréia em cobertura e via foliar em feijoeiro, Piracicaba. Scientia Agrícola, 57: 293-298.

Andrews, D. J.; Hanna, W. W.; Rajewski, J.; Collins, V. P. 1996. Advance ingrain pearl milhet: utilization andproduction research. In: Janick, J. (Ed.). Progress in new crops. ASHSPress, Alexandria, Egito, p.170-177.

Bonamigo, L. A. 1999. A cultura do milheto no Brasil, implantação e desenvolvimento no cerrado. Anais do Workshop Internacional de Milheto, Brasília, Brasil, p.31-65.

Carvalho, N. M.; Nakagawa, J. 1980. Sementes: ciência, tecnologia e produção. Fundação Cargill, Campinas, Brasil, 326pp.

Carvalho, N. M.; Nakagawa, J. 2000. Sementes: ciência, tecnologia e produção. FUNEP, Jaboticabal, Brasil, 588pp.

Costa, N. L. 1992. Estabelecimento, formação e manejo de pastagens de milheto. Lavoura, 45: 7-12.

Degenhart, N. R.; Werner, B. K.; Burton, G. W. 1995. Forage yield and quality of a brown mid-rib mutant in pearl millet. Crop Science, 35: 986-988.

Hanna, W. W.; Wright, D. 1995. Planting date, resust, and cultivar maturity effects on agronomic characteristics of pearl millet. In: Teare, I. D. (Ed.). National grain pearl millets. University of Georgia, Athens, USA, p.28-31.

Heringer, I. 1995. Efeito de níveis de nitrogênio sobre a dinâmica de uma pastagem de milheto (Pennisetum americanum (L.) Leek) sob pastejo. Dissertação de Mestrado, Universidade de Santa Maria, Brasil, 133pp.

Kichel, A. N.; Macedo, M. C. M. 1996. Milheto, a opção forrageira para alimentar animais na época seca. A Lavoura, 99: 20-21.

Pitol, C.; Borges, E.P.; Broch, D. L.; Siede, P. K.; Erbes, E. J.; Chirata, I. N. 1996. O milheto na integração agricultura pecuária. In: Casale, H. (Ed.). Café adensado plantio: um desafio e números o que vale é a diferença. Potafós, Piracicaba, Brasil, 89pp.

Netto, D. A. M. 1998. A cultura do milheto. Embrapa Milho e Sorgo, Sete Lagoas, Brasil, 6pp. (Comunicado Tecnico, 11).

Rodrigues, M. B.; Kiehl, J. C. 1986. Volatilização de amônia após emprego de uréia em diferentes doses e modos de aplicação. Revista Brasileira de Ciência do Solo, 10: 37-43. 